\title{
The new injury severity score: A more accurate predictor of need ventilator and time ventilated in trauma patients than the injury severity score
}

\author{
Azim Honarmand, Mohammadreza Safavi
}

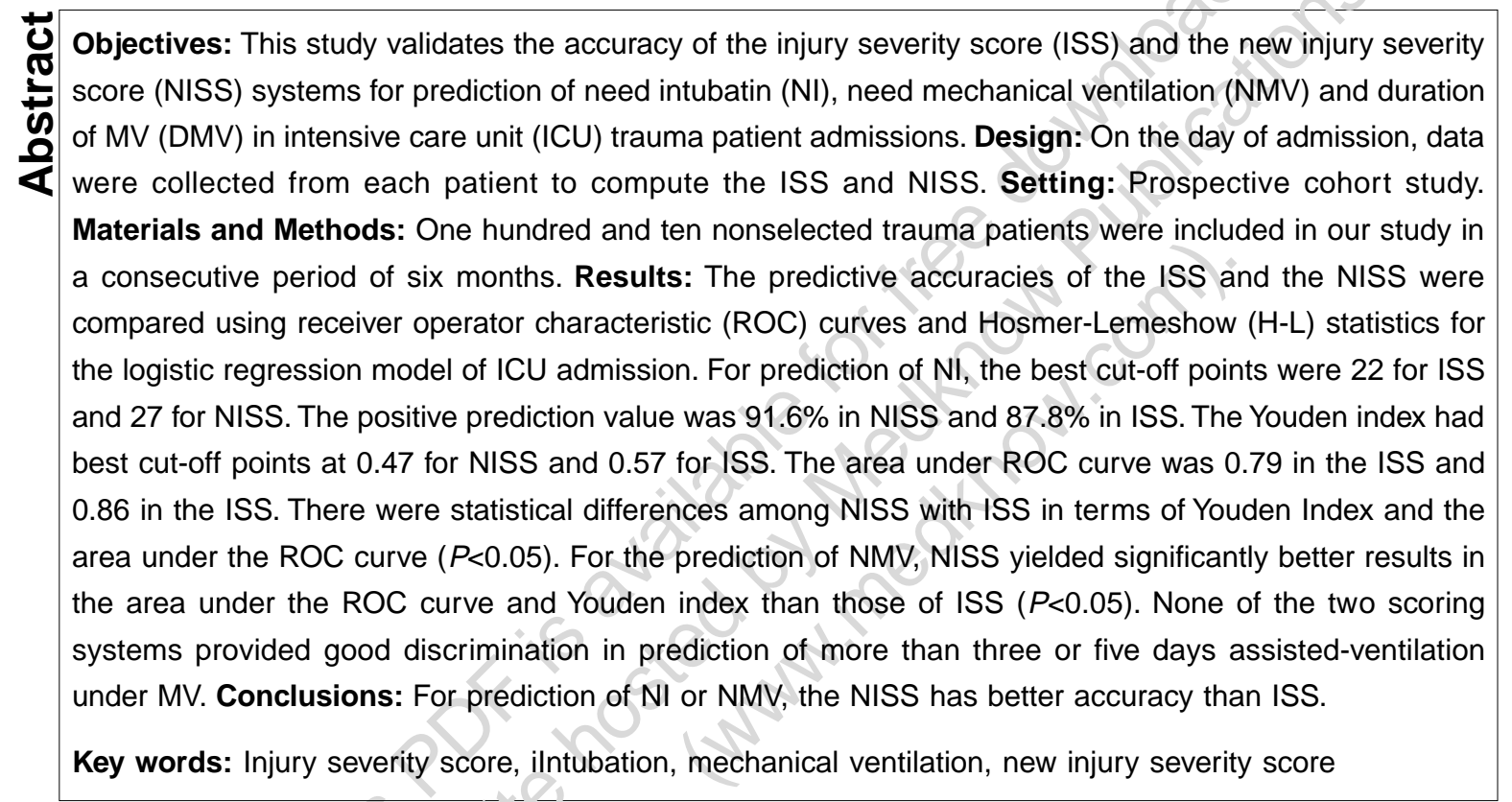

Many different clinical conditions can lead to respiratory failure and mechanical ventilation. ${ }^{[1]}$ The length of time a patient remains on mechanical ventilation depends on the cause and severity of respiratory failure. ${ }^{[2]} A$ common difficulty in the intensive care unit (ICU) is predicting the weanability of patients following prolonged mechanical ventilation. ${ }^{[3]}$ Many clinically measured parameters and calculated laboratory variables have been investigated

\section{From:}

Department of Anesthesiology and Intensive Care, School of Medical Science, Isfahan University, Isfahan, Iran

\section{Correspondence:}

Dr. Mohammadreza Safavi, Department of Anesthesia and Intensive Care, School of Medical Science, Isfahan University, Isfahan, Iran

E-mail: safavi@med.mui.ac.ir as possible predictors of mechanical ventilation dependency and outcome..$^{[3]}$ Measurements reflecting lung mechanics (e.g., tidal volume, lung and chest wall compliance), the strength of respiratory muscles (e.g. peak inspiratory pressure) and gas-exchanging capability (e.g., $\mathrm{PaCO}_{2}$ ) have been proposed to predict the success of discontinuing mechanical ventilation. ${ }^{[4,5]}$ Although pulmonary function tests are important in determining mechanical ventilation dependency, other components of the clinical situation need to be considered when attempting to wean a patient from mechanical ventilation. ${ }^{[3]}$ IIIness severity scoring systems have become important tools for the study of patient outcomes relating to withdrawal of mechanical ventilation. ${ }^{[2,6-8]}$ The 
direct correlation between these scores and duration of ventilation may initially surprising, since they do not specifically include pulmonary function tests or weaning parameters. There appear to be several reasons for this correlation and several advantages to incorporating general severity of illness score as part of a comprehensive approach to predicting duration of mechanical ventilation: First, they include many respiratory physiologic variables, such as respiratory rate, $\mathrm{P}(\mathrm{A}-\mathrm{a}) \mathrm{O}_{2}, \mathrm{PaO}_{2}$ and $\mathrm{PaCO}_{2}$ that are known to be influential determinant of successful weaning. ${ }^{\left[{ }^{[]}\right.}$Second, they include nonrespiratory variables, such as a neurologic assessment and chest wall or lung injury scale which influence the duration of ventilation. The impact of these nonrespiratory variables on the duration of ventilation is considerable, may be underestimated by physicians and is not included in many traditional weaning indexes. ${ }^{[5]}$ Finally, these scores, while not requiring specific pulmonary function testing, dose provide an objective summary measure of many respiratory and nonrespiratory variables that are important to weaning and mortality risk and thus duration of ventilation. On the other hand, ICU patients requiring mechanical ventilation have a high mortality and consume disproportionate amount of nursing and financial resources. ${ }^{[10,11]}$ The ability to systemically predict the duration of ventilation for a general ICU population would also be useful for examining resource allocation, designing and evaluating clinical trials and as a mean for analyzing practice pattern among ICUs. ${ }^{[9]}$ Since its creation in 1974 by Baker et $a^{{ }^{12]}}$ the injury severity score (ISS) has been considered the "gold standard" in anatomic injury severity indicators. ISS is used to describe trauma populations, to evaluate the quality of trauma care ${ }^{[13,14]}$ and to control for case mix in trauma research. ${ }^{[15]}$ ISS sums the severity score for the three most severe injuries, but it only considers one injury per body region. Therefore, one can suspect that the ISS underscores the severity in trauma victims with multiple injuries confined to one body region. ${ }^{[16]}$ Perhaps the most important drawback of the ISS cannot be addressed with statistical techniques: the ISS only considers one injury in each body region. This leads to injuries being overlooked and to less severe injuries occurring in other body regions being included in the calculation over more serious ones in the same body region. ${ }^{[17]} A$ simple modification to the ISS, the new injury severity score (NISS), was designed by Osler et al in 1997 to counter this problem. ${ }^{[13]}$ The NISS is simply the sum of squares of the three most severe injuries, regardless of body region injured. Therefore, the NISS will be equal to or higher than the ISS. Lavoie et al ${ }^{[18]}$ study showed that significantly more patients required prolonged mechanical ventilation (PMV) if they had any one of the following: Swan-Ganz, ISS more than $20, \mathrm{PaO}_{2} / \mathrm{FiO}_{2}$ less than 250 or fluid retention more than $2000 \mathrm{cc}$ at $48 \mathrm{~h}$. Despite the fact that the ISS was designed to predict death, it is also used to model many other outcomes such as hospital stay, ICU stay, time ventilated, complications and emergency surgery. ${ }^{[19]}$ There were no studies to evaluate NISS in predicting duration of mechanical ventilation and investigate any potential advantages of NISS over ISS in this relation. The purpose of this study was to determine whether the ISS or NISS is a predictor of need-ventilator and time-ventilated in general and in subgroups of trauma patients according to age, penetrating trauma and body region injured.

\section{Materials and Methods}

The database consisted of prospectively identified, consecutive trauma patient admissions in Alzahra hospital. Between May 2005 and October 2005, all consecutive trauma admissions during the six month period with age older than 16 years and survival greater than $24 \mathrm{~h}$ were entered into the study. For the purpose of this study, patients who suffered burn trauma, patients who were dead on arrival at the emergency department or patients with isolated hip fractures, because these constitute a unique population of patients with a high probability of death, were excluded. The Institutional Review Board at university approved the study. abbreviated injury scale (AIS) coding is performed by a trained physician from patients' medical files. This parameter takes values from 1 to 75 and is computed from AIS severity values, which vary from 1 to 6 . The ISS is the sum of squares of the largest AIS severity value from each of the three most severely injured body regions. Any patient with an AIS value of 6 is automatically scored 75 on the ISS scale. NISS values were computed as the sum of squares of the three highest AIS values, regardless of body region. During ICU stay, patients' need intubation or assisted ventilation and duration of mechanical ventilation were evaluated. The ISS, the NISS, partial arterial oxygen tension $\left(\mathrm{PaO}_{2}\right)$ / inspired fraction of oxygen $\left(\mathrm{FiO}_{2}\right)$, net fluid balance fluid (retention more or less than $2000 \mathrm{cc}$ ), calculated during 
$48 \mathrm{~h}$ after surgical intensive care unit admission. The objective criteria for ventilator discontinuation readiness were defined as ${ }^{[1]}$ passage of the spontaneous breathing trial (SBT] screen and ${ }^{[2]}$ successful completion of a 30 min SBT performed on flow-by mode, $\mathrm{PS} \leq 8 \mathrm{~cm} \mathrm{\textrm {H } _ { 2 }} \mathrm{O}$ on $P E E P \leq 8 \mathrm{~cm} \mathrm{H}_{2} \mathrm{O}$ or T piece. Successful discontinuation of mechanical ventilation was defined as continuous independence from ventilator support for a $24 \mathrm{~h}$ period. ${ }^{[20]}$

\section{Data analysis}

The sensitivity, specificity and correct prediction of outcome for each cut-off point were calculated in MedCalc $^{\circledR}$ version 9.0.1.1.for ISS and NISS. The best cut-off point in each scoring system is determined when the point yields the best specificity and sensitivity in the two-by-two table. The best Youden index also determines the best cut-off point. The Youden index is used to compare the proportion of cases correctly classified. The higher the Youden index ${ }^{[21]}$ the more accurate is the prediction (higher true positive and true negatives and fewer false positive and false negatives) at the cut-off point. Descriptive statistics were expressed as mean \pm SD unless otherwise stated. A receiver operating characteristic (ROC) curve ${ }^{[22]}$ depicts the relation between true positive and false positive for each scoring system. This method compares scores without fixing arbitrary cut-off points. The ROC curve is calculated by the MedCalc ${ }^{\circledR}$ version 9.0.1.1. The area under the ROC curve is evaluated. Such an area represents the probability that a randomly chosen diseased subject is more correctly rated or ranked than a randomly chosen nondiseased subject. ${ }^{[22]}$ A value of 0.5 under the ROC curve indicates that the variable performs no better than chance and a value of 1.0 indicates perfect discrimination. A larger area under the ROC curve represents more reliability ${ }^{[23]}$ and good discrimination of the scoring system. The value of the Hosmer-Lemeshow statistic was obtained by SPSS 11.0. Values of more than 15.5 represent poor agreement of calibration between the outcomes estimated from the model and the observed outcomes. Lower chi-square values and higher $P$ values are associated with a better fit. A good fit was defined as $P>0.05$.

\section{Results}

One hundred ten trauma patients were admitted into our ICU in a 10 month period. Their ages varied from16 to 87 years with a mean of $34.65 \pm 16.34$ years. There were 93 males and 17 females [Table 1]. Of all trauma patients enrolled in the study, blunt trauma accounted for $90.9 \%$ of the admissions. The patients also were grouped according to region injured: 1) head (skull, face and neck); 2) torso (chest, abdomen and pelvic content); and 3) limbs including pelvic girdle. Sixty-two (56.4\%) patients needed intubation in ICU; fifty (45.5\%) patients needed respiratory support under mechanical ventilation (MV). Among the intubated patients, 50 (80.6\%) patients were supported by MV. Admission ISS and NISS scores (0-24h) were significantly higher in patients need intubation ( $\mathrm{NI}$ ) or mechanical ventilation (NMV). The distribution of scores on day 1 and probability of need intubation (NI) or mechanical ventilation (NMV) and duration of mechanical ventilation (DMV) or intubation (DI) derived from each scoring system are shown in [Figures 1-3]. The sensitivity, specificity, correct prediction outcome, Youden index and area of the ROC curve at the best cutoff point for $\mathrm{NI}$ or NMV are presented in Tables 2 and 3. There are statistical differences in Youden index and area under the ROC curve between NISS with ISS for NI or NMV. In both $\mathrm{NI}$ and NMV, the NISS provides good results, as shown in Tables 2 and 3. By contrast, ISS, yield poor results. Therefore, NISS plays a crucial role in the prediction of NI or NMV. None of the three scoring systems provide good
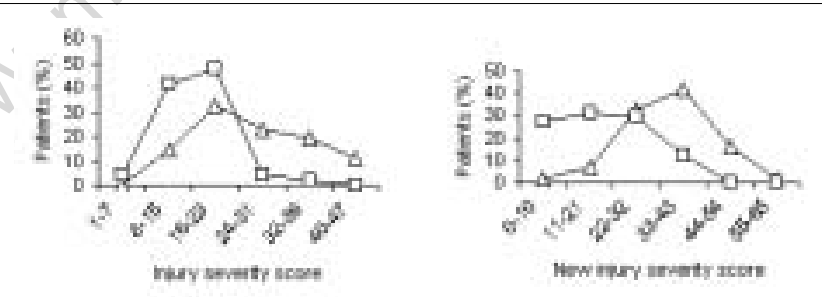

Figure 1: Distribution of injury severity score (ISS) and new injury severity score (NISS) in the study patients and need intubation in each category. The higher the scores in ISS or NISS, the numbers of intubated patients were more than nonintubated. $\Delta=$ intubated; $\Delta$ $=$ not intubated

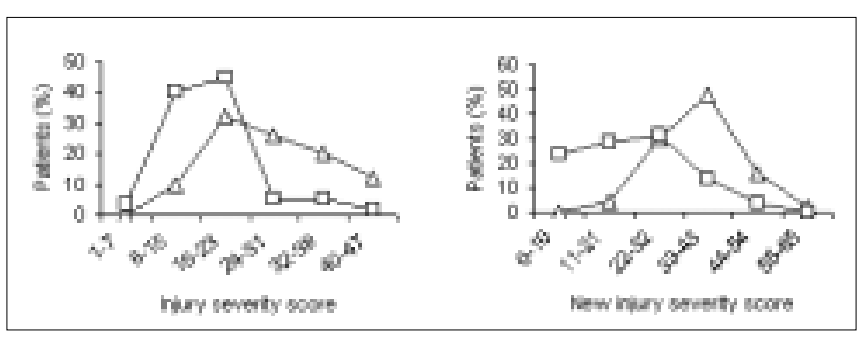

Figure 2: Distribution of injury severity score (ISS) and new injury severity score (NISS) in the study patients and need mechanical ventilation (NMV) in each category. The higher the scores in ISS or NISS, the numbers of patients under mechanical ventilation were more than them without ventilatory support. $\Delta=\mathrm{NMV} ; \mathrm{\square}=$ no NMV 


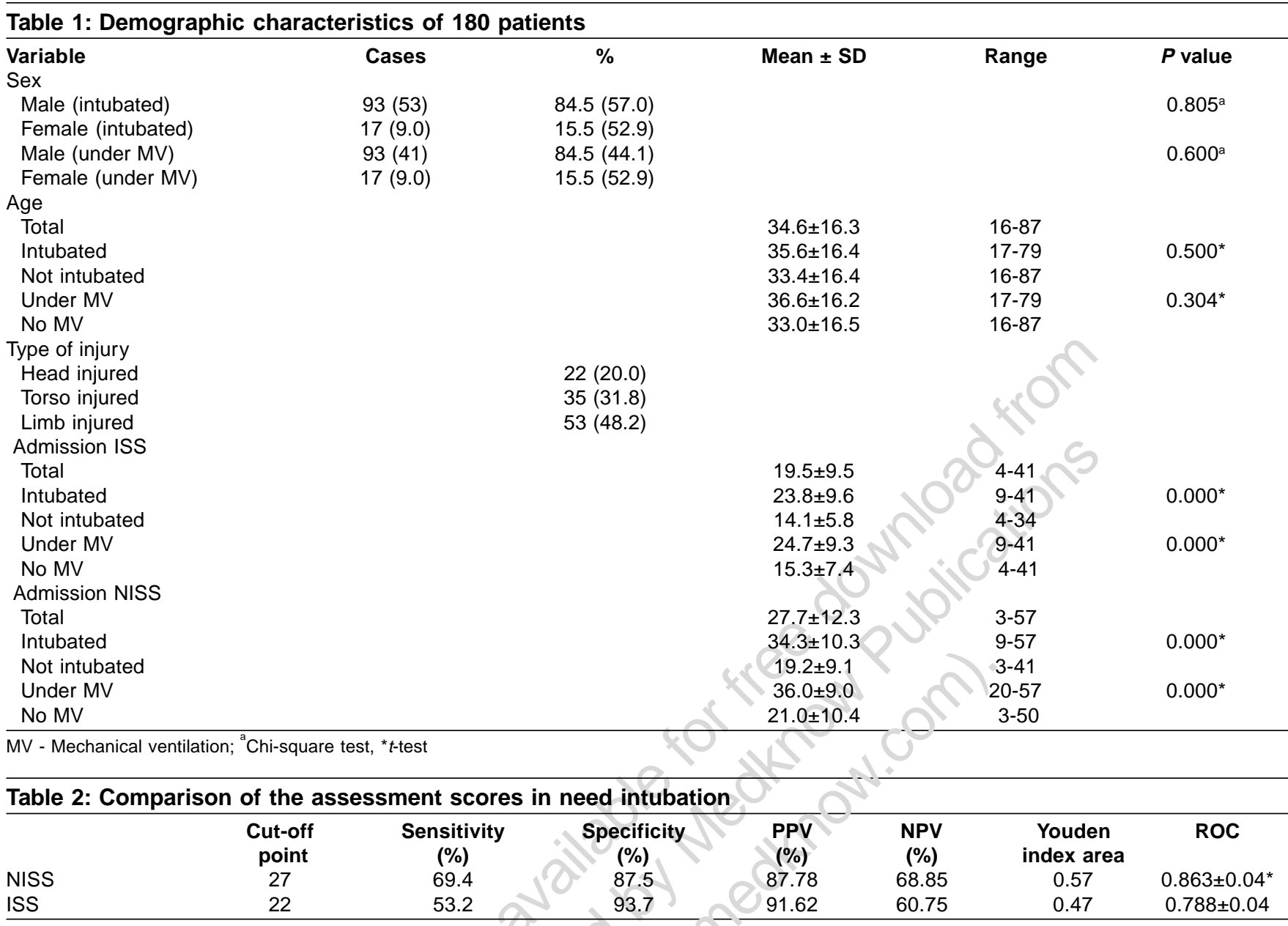

ISS = Injury severity score; NISS = New injury severity score; PPV = Positive predictive value; NPV = Negative predictive value, ${ }^{*}$ NISS vs ISS: $P<0.05$

Table 3: Comparison of the assessment scores in need mechanical ventilation

\begin{tabular}{lccccccc}
\hline & $\begin{array}{c}\text { Cut-off } \\
\text { Point }\end{array}$ & $\begin{array}{c}\text { Sensitivity } \\
(\%)\end{array}$ & $\begin{array}{c}\text { Specificity } \\
(\%)\end{array}$ & $\begin{array}{c}\text { PPV } \\
\text { (\%) }\end{array}$ & $\begin{array}{c}\text { NPV } \\
(\%)\end{array}$ & $\begin{array}{c}\text { Youden } \\
\text { index area }\end{array}$ & $\begin{array}{c}\text { ROC } \\
\text { NISS }\end{array}$ \\
\hline ISS & 27 & 76.0 & 81.7 & 77.60 & 80.30 & 0.58 & $0.861 \pm 0.04^{*}$ \\
\hline
\end{tabular}

ISS = Injury severity score; NISS = New injury severity score; PPV = Positive predictive value; NPV = Negative predictive value, ${ }^{*}$ NISS vs ISS: $P<0.05$

discrimination in prediction of more than three or five days respiratory support under MV $(A \cup C<0.5)$. The calibration of the NISS system is adequate for prediction of $\mathrm{NI}$ or NMV, as shown in Figure 4.

\section{Discussion}

In comparing the NISS with the ISS, we find the accuracy of the NISS is significantly better than that of the ISS for prediction of need intubation or mechanical ventilation. The NISS is certainly a more logical choice of anatomic trauma severity measure. ${ }^{[24,25]}$ For example, a patient with three injuries to the head/neck or spine, all with AIS values of 5, will have an ISS value of 25 but an NISS value of 75 . Meanwhile, a patient with AIS values of 5 in three different body regions will have both an ISS and NISS value of 75 .
A comparison of the two patients based on their ISS would therefore be inaccurate. ${ }^{[19]}$ This study demonstrates that in addition to making more clinical sense, the NISS is also a more accurate choice from a statistical standpoint. The NISS is not much better than the ISS in the prediction of duration of MV, because many biases are found in the use of these systems. First, treatment error is not predictable, especially in surgical patients. ${ }^{[26]}$ Second, the data collected on the day of admission may not reflect completely the unforeseen events which may be major determinants of outcome. ${ }^{[27]}$ Third, the co-morbidity condition $^{[28]}$ is not taken into account enough in these systems. There are severs potential uses for the relations on predicting $\mathrm{NI}$ or NMV. First, the equation provides a new tool for quality assessment and improvement. ${ }^{[9]}$ 


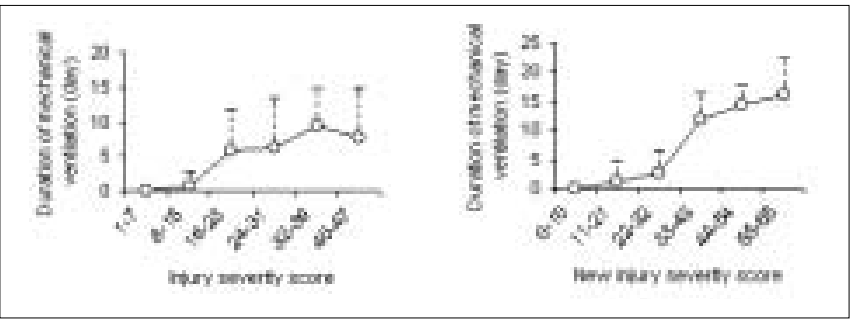

Figure 3: Distribution of injury severity score (ISS) and new injury severity score (NISS) in the study patients and duration of mechanical ventilation (DMV) in each category. The higher the scores in ISS or NISS, the higher the ventilator days. Data are mean \pm SD

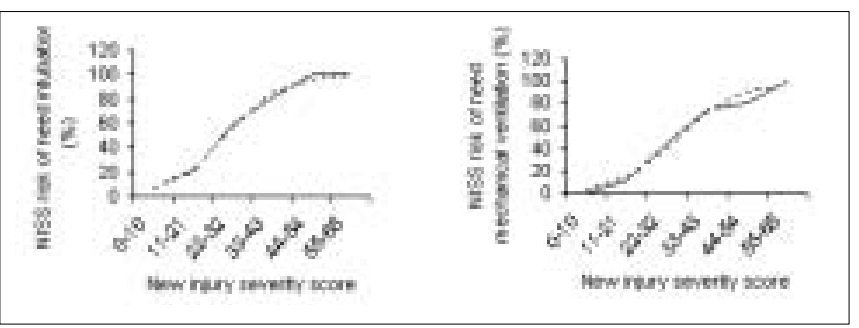

Figure 4: The calibration of NISS system on our patients for the observed and predictive risk of need intubation and need mechanical ventilation; = predicted; = observed. NISS = new injury severity score; $\mathrm{H}-\mathrm{L}=$ Hosmer-Lemeshow.

Mechanical ventilation constitutes one of the greatest personnel, resource and financial commitments for most critical care units, but, to date and to our knowledge, there has not been an adequate method for ICU managers to assess ventilator utilization or compare their unit's practices to those of other ICUs after adjusting for differences in patients treated..$^{[9]}$ If an ICU has more patients requiring intubation or ventilator and longer duration of ventilation than predicted over a prolonged period, this may represent sufficient evidence for a study of the unit's ventilator practice. Such an internal study might discover that patients in certain diagnostic categories are routinely ventilated longer than similar patients in the other units. Alternatively, the study might discover opportunities for streamlining or improving ventilatory practices. The ICU manager could then institute quality improvement measures, e.g., establish improved weaning protocol and use future analyses to document the impact of those changes. ${ }^{[29,30]}$ Patients undergoing prolonged (seven days or more) ventilation represented $52.6 \%$ of all ventilated patients and $18.2 \%$ of all ICU admission in our study. Such patients consume a disproportionate amount of technological and financial resources and reimbursement is frequently incomplete. Several studies have reported the success of intermediate care units, noninvasive respiratory units, long-term weaning facilities and multidisciplinary "weaning teams" in maintaining quality of care at a substantial cost saving and less resource use. ${ }^{[11,32]}$ Further research is needed but our study may have an application in helping to identify a selected group of ventilated patients with a predicted long duration of ventilation (i.e., > seven days) who might benefit from wean team consult or early consideration for transfer to a subacute facility. To our knowledge, this study is the first to date to investigate the question of whether NISS more accurately predicts need intubation on mechanical ventilation than the ISS. One flaw of this study is associated with the well-known lack of data precision, which plagues all trauma registries. ${ }^{[33]}$ Most importantly, coding errors could affect the accuracy of the AIS values assigned. Physicians cannot be blinded to patients' status. It is therefore possible that they may pay more attention to AIS coding of intubated than of nonintubated. This bias would lead to an underestimation of injury severity among intubated compared with non-intubated and would artificially increase the accuracy of AIS-based severity measures in predicting outcome. Because ISS/NISS values are based on the same AIS codes, however, this bias should not affect the comparison of the two measures. It would also be interesting to verify whether the differences in NISS/ISS predicting power hold up for Level II or III trauma centers and to perform analyses according to the presence or absence of comorbidities. In conclusion, we have shown that the NISS outperforms the ISS in predicting NI or NMV. One result of our study has been an emphasis on better outcome analysis and improved cost-efficiency. We recommend that future studies adopt the NISS for controlling bias caused by trauma severity case mix but that particular attention be paid to the form of the variable introduced into regression models. When predicting $\mathrm{NI}$ or NMV from trauma, the importance of investigating the contribution of other risk factors such as age, comorbidity, physiologic indicators, injury mechanism and body area injured should not be overlooked. This work is preliminary, but we believe that, because of these results, further efforts to define outcome- documentation, analyze practice pattern and improve cost-efficiency for mechanical ventilation and other high-cost technologies common to ICUs are warranted.

\section{References}

1. Balk R, Bone RC. Classification of acute respiratory failure. Med Clin North Am 1983;67:551-6.

2. Gillespie DJ, Marsh HM, Divertie MB, Meadows JA $3^{\text {rd }}$. Clinical outcome of respiratory failure in patients requiring prolonged 
(more than 24 hours) mechanical ventilation. Chest 1986;90:364-9.

3. Sporn PH, Morganroth ML. Discontinuation of mechanical ventilation. Clin Chest Med 1988;9:113-26.

4. Jabour ER, Rabil DM, Truwit JD, Rochester DF. Evaluation of a new weaning index based on ventilatory endurance and the efficiency of gas exchange. Am Rev Respir Dis 1991;144:531-7.

5. Yang KL, Tobin MJ. A prospective study of indexes predicting the outcome of trials of weaning from mechanical ventilation. $\mathrm{N}$ Engl J Med 1991;324:1445-50.

6. Diringer MN, Edwards DF, Aiyagari V, Hollingsworth H. Factors associated with withdrawal of mechanical ventilation in a neurology/neurosurgery intensive care unit. Crit Care Med 2001;29:1792-7.

7. Sapijaszko MJ, Brant R, Sandham D, Berthiaume $\mathrm{Y}$. Nonrespiratory predictor of mechanical ventilation dependency in intensive care unit patients. Crit Care Med 1996;24:601-7.

8. Ross BJ, Barker DE, Russell WL, Burns RP. Prediction of long-term ventilatory support in trauma patients. Am Surg 1996;62:19-25.

9. Seneff MG, Zimmerman JE, Knaus WA, Wagner DP, Draper EA. Predicting the duration of mechanical ventilation. The importance of disease and patient characteristics. Chest 1996;110:469-79.

10. Strauffer JL, Fayter NA, Graves B, Cromb M, Lynch JC, Goebel $P$. Survival following mechanical ventilation for acute respiratory failure in adult men. Chest 1993;104:1222-9.

11. Wagner DP. Economics of prolonged mechanical ventilation. Am Rev Respir Dis 1989;140:S14-8.

12. Boyd CR, Tolson MA, Copes WS. Evaluating trauma care: The TRISS methodology. J Trauma 1987;27:370-8.

13. Husum H, Strada G. Injury Severity Score versus New Injury Severity Score for penetrating injuries. Prehospital Disaster Med 2002;17:27-32.

14. Champion HR, Copes WS, Sacco WJ, Lawnick MM, Keast SL, Bain LW Jr, et al. The Major Trauma Outcome Study: establishing national norms for trauma care. J Trauma 1990;30:1356-65.

15. Fulda GJ, Tinkoff GH, Giberson F, Rhodes M. In-house trauma surgeons do not decrease mortality in a level I trauma center. J Trauma 2002;53:494-500.

16. Baker SP, O'Neill B, Haddon W Jr, Long WB. The injury severity score: A method for describing patients with multiple injuries and evaluating emergency care. J Trauma 1974;14:187-96.

17. Stevenson M, Segui-Gomez M, Lescohier I, Di Scala C, McDonald-Smith $\mathrm{G}$. An overview of the injury severity score and the new injury severity score. Inj Prev 2001;7:10-3.

18. Lavoie A, Moore L, Lesage N, Liberman M, Sampalis JS. The Injury Severity Score or the New Injury Severity Score for predicting intensive care unit admission and hospital length of stay? Injury 2005;36:477-83.

19. Lavoie A, Moore L, LeSage N, Liberman M, Sampalis JS. The new injury severity score: A more accurate predictor of in-hospital mortality than the injury severity score. J Trauma 2004;56:1312-20.

20. Marelich GP, Murin S, Battistella F, Inciardi J, Vierra T, Roby M.
Protocol weaning of mechanical ventilation in medical and surgical patients by respiratory care practitioners and nurses: Effect on weaning time and incidence of ventilator-associated pneumonia. Chest 2000;118:459-67.

21. Youden WJ. Index for rating diagnostic test. Cancer 1950;3:32-5.

22. Hanley JA, McNeil BJ. The meaning and use of the area under a receiver operating characteristic (ROC) curve. Radiology 1982;143:29-36.

23. Oh TE, Hutchinson R, Short S, Buckley T, Lin E, Leung D. Verification of the acute physiology and chronic evaluation scoring system in a Hong Kong intensive care unit. Crit Care Med 1993;21:689-705.

24. Osler T, Baker SP, Long W. A modification of the injury severity score that both improves accuracy and simplifies scoring. $J$ Trauma 1997;43:922-6.

25. Brenneman FD, Boulanger BR, McLellan A, Redelmeier DA. Measuring injury severity: Time for a change? J Trauma 1998;44:580-2.

26. Meyer AA, Messick WJ, Young P, Baker CC, Fakhry S, Muakkassa $\mathrm{F}$, et al. Prospective comparison of clinical judgment and APACHE II score in predicting the outcome in critically ill surgical patients. J Trauma 19992;32:747-54.

27. Le Gall JR, Loirat P, Alperovitch A, Glaser P, Granthil C, Mathieu $D$, et al. A simplified acute physiology score for ICU patients. Crit Care Med 1984;12:975-7.

28. Knaus WA, Wagner DP, Draper EA, Zimmerman JE, Bergner M, Bastos PG, et al. The APACHE III prognostic system: Risk prediction of hospital mortality for critically ill hospitalized adults. Chest 1991;100:1619-36.

29. Cohen IL, Lambrinos J, Fein IA. Mechanical ventilation for the elderly patient in intensive care: Incremental changes and benefits. JAMA 1993;269:1025-9.

30. Kritchevsky SB, Simmons BP. Continuous quality improvement: concepts and applications for physician care. JAMA 1991;266:1817-23.

31. Krieger BP, Ershowsky P, Spivack D, Thorstenson J, Sackner MA. Initial experience with a central respiratory monitoring unit as a cost saving alternative to the intensive care unit or medicare patients who rquire long-term ventilator support. Chest 1988;93:395-7.

32. Cohen IL, Bari N, Strosberg MA, Weinberg PF, Wacksman RM, Millstein $\mathrm{BH}$, et al. Reduction of duration and cost of mechanical ventilation in an intensive care unit by use of a ventilatory management team. Crit Care Med 1991;19:1278-84.

33. Owen JL, Bolenbaucher RM, Moore ML. Trauma registry databases: A comparison of data abstraction, interpretation and entry at two level I trauma centres. J Trauma 1999;46:1100-4.

Source of Support: Nil, Conflict of Interest: None declared. 